\title{
Predatory Fish Select for Coordinated Collective Motion in Virtual Prey
}

\author{
C. C. Ioannou, ${ }^{1,2 *}$ V. Guttal, ${ }^{1,3}$ I. D. Couzin ${ }^{1 *}$
}

Movement in animal groups is highly varied and ranges from seemingly disordered motion in swarms to coordinated aligned motion in flocks and schools. These social interactions are often thought to reduce risk from predators, despite a lack of direct evidence. We investigated risk-related selection for collective motion by allowing real predators (bluegill sunfish) to hunt mobile virtual prey. By fusing simulated and real animal behavior, we isolated predator effects while controlling for confounding factors. Prey with a tendency to be attracted toward, and to align direction of travel with, near neighbors tended to form mobile coordinated groups and were rarely attacked. These results demonstrate that collective motion could evolve as a response to predation, without prey being able to detect and respond to predators.

$\mathrm{F}$ rom herding ungulates to shoaling fish, nesting birds, and swarming crickets, animals living in groups are generally less at risk from predators (1). Mechanisms include the ability of groups to detect predators sooner and from a greater distance (the "many eyes" effect) and cognitive confusion of the predator, caused by having to choose among many possible targets 
(1). Studies have focused on the costs and benefits of group size and position within the group $(2-4)$, but how predation risk varies with the response of individuals to their neighbors is not well understood because of difficulties in its measurement $(5,6)$ and manipulation (7). One such behavioral response that is common in nature is the tendency for individuals to align their direction of travel with that of near neighbors, forming coordinated "polarized" groups. It is often assumed that coordination between prey makes them harder to catch by enhancing information transfer between individuals $(5,6)$ or by increasing the confusion effect, although little experimental work supports this idea $(7,8)$.

How animals move together has been simulated by agent-based models with generic behavioral tendencies: repulsion when neighbors are too close and otherwise aligning with, and/or being attracted to, neighbors. These models are able to recreate group movement such as swarms, in which attraction dominates alignment tendency, and coordinated polarized flocks or schools, in which alignment tendency becomes relatively strong (9-13). Although studies link this mechanistic approach back to functional explanations (14-16), there are few that have explicitly dealt with the relationship between predation and dynamic group behavior. Models can recreate the macroscopic responses of prey when under threat (17) and the evolution of swarmlike or highly polarized, coordinated groups as a direct result of simulated predator behavior (18). In many cases, however, it is unclear which properties of prey behavior are being selected for and why, and it remains to be established whether any real-life predators select for coordinated motion.

To explore these issues, we investigated how a predatory fish, the bluegill sunfish (Lepomis macrochirus), hunts simulated prey. Bluegills are generalist predators whose body plan is specialized for hunting in complex vegetated environments (19). Our system allows us to isolate the specific selection pressure of predation risk from the multitude of factors influencing the evolution of any trait, such as the associated costs of the trait and taxonomic constraints $(20)$. In nature there is a wide variety of prey responses to predators; some react to predators as they approach (2), whereas others only respond once an attack is made (21), and these responses are further influenced by factors such as prey group size $(1,21)$. Because our simulated prey could not respond to the fish, we analyzed only the first attack from each fish, which is analogous to situations where risk is determined primarily by the

${ }^{1}$ Department of Ecology and Evolutionary Biology, Princeton University, Princeton, N] 08544, USA. ${ }^{2}$ School of Biological Sciences, University of Bristol, Woodland Road, Bristol BS8 1UG, UK. ${ }^{3}$ Centre for Ecological Sciences, Indian Institute of Science, Bangalore 560012, India.

*To whom correspondence should be addressed. E-mail: C.C.Ioannou@bristol.ac.uk (C.C.I.); icouzin@princeton.edu (I.D.C.) first attack (22). A simulation of animal movement (23) consisting of a behaviorally heterogeneous population of 16 prey was projected onto a translucent screen on an inner side of the test tank (Fig. 1 and fig. S1). Individual prey behavior was encoded by three traits: the strength of their behavioral tendencies to be attracted toward $\left(\omega a_{\mathrm{i}}\right)$, orientate direction of travel with $\left(\omega o_{\mathrm{i}}\right)$, or ignore $\left(\omega p_{\mathrm{i}}\right)$ near neighbors [the three traits were normalized so that their sum was $1(12,24,25)$ (table S1)]. Depending on these traits, prey exhibited a range of movement behaviors, including solitary random walk, formation and maintenance of aggregations, and coordinated polarized motion (movie S1).

The predators exerted a strong selection pressure on the virtual population, with some prey being attacked often while others were never attacked (Fig. 2A). The risk of being targeted was minimized for individuals with characteristics that balanced both attraction and orientation, resulting in an interaction between these two parameters [generalized linear model (GLM): likelihood ratio test $\left.\left(\mathrm{LRT}_{1,12}\right)=11.43, P=0.00072\right]$. This suggests that to most effectively avoid predation, prey should both move toward and align with their near neighbors, behavior that generates moving groups of coordinated individuals (12).

To a large degree, the strength of attraction mediates the group size for the simulated prey; when attraction is zero, prey are most frequently found alone (Fig. 2B). Although increasing the orientation parameter has only a minor effect on group size, it increases the straightness (i.e., decreases the "curvedness" or tortuosity) of the prey's path substantially when that prey is in a group (Fig. 2C). For example, although the prey type with $\omega a_{\mathrm{i}}=0.2$ and $\omega o_{\mathrm{i}}=0.6$ is rarely solitary, it tends to exhibit relatively directed, lowtortuosity motion (Fig. 2C), similar to the prey type that has no social tendency $\left(\omega a_{\mathrm{i}}=0.0\right.$ and $\left.\omega o_{\mathrm{i}}=0.0\right)$ and is rarely found in a group. Furthermore, the tortuosity of a prey individual's path typically scales negatively with their group's polarization (the directional coherence among group members), so that nonsolitary individuals with low-tortuosity paths are typically in groups with high polarization (fig. S2).

To explore how these behaviors mediated the effects of both attraction and orientation on risk, we could not simply correlate the mean group size or tortuosity of a prey type with the number of attacks it received, because any choice made by the predator is constrained by the other prey phenotypes present at the time of attack. Instead, we created a null predator that chose a prey randomly at the same time steps as the observed attacks and compared the relationship between the targets' tortuosity and the group size expected from random targeting to that actually observed from the real predatory fish (23). Compared to random targeting, the fish disproportionately targeted prey in smaller groups, and this was strongest when prey were also taking less-tortuous paths (figs. S3 and S4). Prey in groups with a coordinated direction of motion (i.e., with high polarization) (12) were at less risk than their counterparts in unpolarized swarms (fig. S3).

Bluegill sunfish employ a characteristic "hovering" behavior during foraging (Fig. 1B) (19), allowing us to approximate the time taken to make each targeting decision. Consistent with a confusion effect (26), this decision time increased with the prey target's group size (GLM: $\left.\mathrm{LRT}_{1,67}=11.32, P=0.00077\right)$. Although this accounts for the targeting of prey in smaller groups, there was no evidence that a prey's tortuosity, either alone or as part of an interaction with group size, had any additional effect [ $\mathrm{LRT}_{1,67}=0.029$, $P=0.86$; and $\mathrm{LRT}_{1,66}=0.22, P=0.64$, respectively; see also $(7,8)]$.

In response to the confusion effect (27), or simply because they are nearer on average $(3,4)$, predators will often attack prey at the edge of groups. To test whether prey relatively far from the group center were attacked disproportionately, we used the null predator procedure described previously. The analysis indicates that this is indeed the case, but only for prey moving with relatively low tortuosity (fig. S5A). Although a number of the behavioral types often moved on paths with low tortuosity (Fig. 2C), prey with high orientation relative to attraction tended to be found more often at the edges of groups (fig. S5, C and D). This "self-assortment" (12) should contribute to selection against such prey types, in addition to their tendency to be solitary (Fig. 2B). However, the edge effect cannot explain why polarized groups were disproportionately less at risk (fig. S3) and why prey types with no tendency
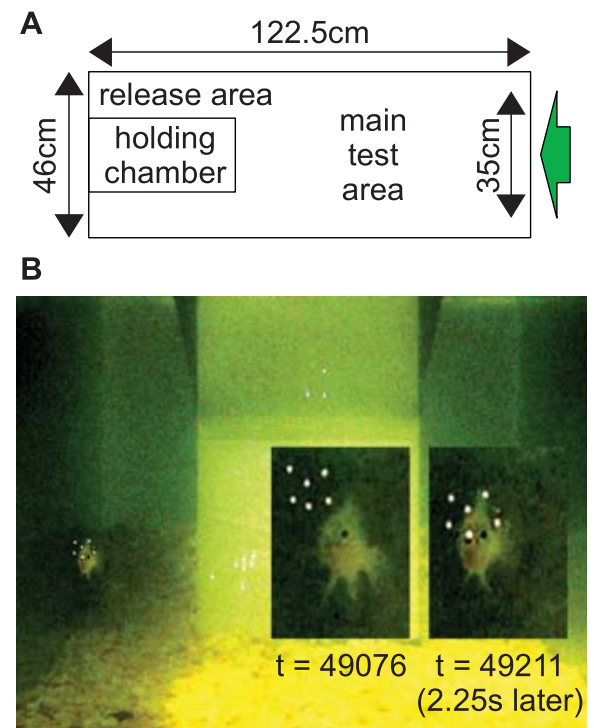

Fig. 1. The experimental system. (A) The simulation was projected (green arrow) onto a screen on the opposite side of the test tank to that where the fish was released. (B) Each attack was preceded by the fish hovering in front of the prey (white dots) (left inset) before accelerating toward a prey, opening the mouth and gill flaps (right inset). $t$, time step in the simulation. 
A

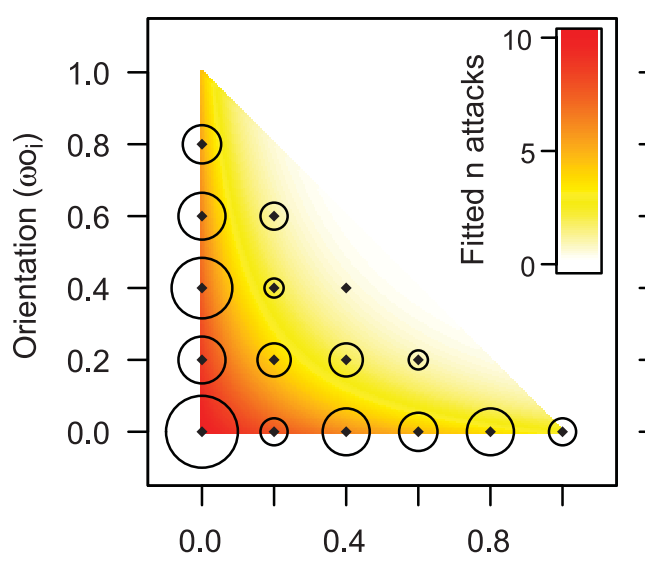

B

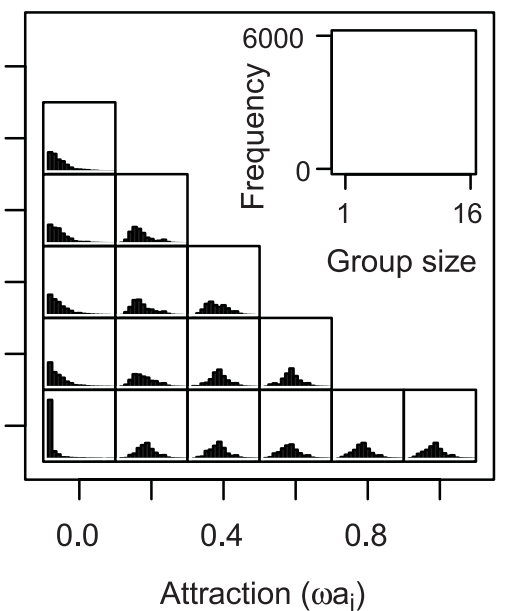

C

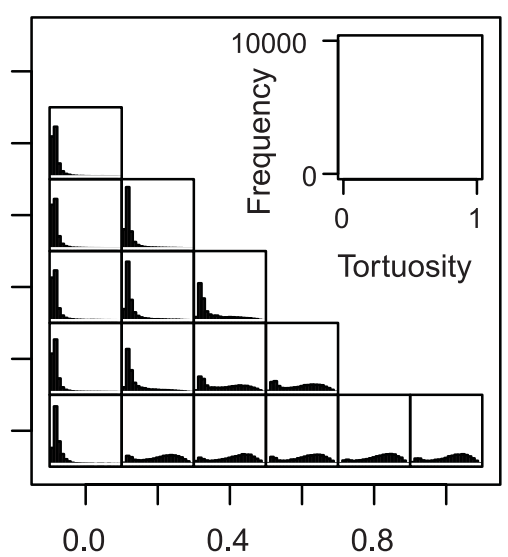

Fig. 2. Risk associated with attraction and orientation traits. (A) Based on their particular attraction and orientation tendencies (black diamonds), the number of attacks from a total of 70 that each prey type received (proportional to the area of the open circles; the largest of these is 14 attacks). The colored gradient represents the fitted values of this relationship from the fully factorial model, where red indicates more attacks and white fewer attacks (see color

scale, inset). (B and C) Frequency distributions of each prey type's group size and tortuosity, respectively, pooled from the simulations presented to the fish in experiment 1 . The scale of the histograms is shown in empty insets at top right. Group size was determined by the number of interconnected prey (23) every 250 time steps, whereas their individual tortuosity was calculated every 100 time steps.

to orient with their neighbors $\left(\omega o_{\mathrm{i}}=0\right)$ were selected against (Fig. 2A).

In complex habitats, such as the littoral zone of lakes where bluegill sunfish are found, both predators and prey often exploit boundaries (2). The boundaries of the projection presented to the predators were periodic; i.e., when prey came in contact with a boundary, they would reappear at the opposite boundary with the same velocity. This ensured that the prey types were found with equal probability anywhere in the projected arena and excluded possible spatial artifacts confounding our results, such as swarming prey being found in the corners (23). Thus, if there is any tendency for prey with certain characteristics to be attacked in particular locations in the projected area, this must be due to the fish's behavior. We found that targeted prey tended to be in larger groups, and to have more tortuous paths, when they were nearer the top and bottom of the projection (Fig. 3; the vertical axis polynomial effect: GLM: $\mathrm{LRT}_{2,65}=$ 13.07, $P=0.0015$; and $\mathrm{LRT}_{2,65}=14.35, P=$ 0.00077 , respectively). When hunting near the edge, predators experience groups that partly, or completely, cross the boundary (from the localized perception of the predator, this is analogous to prey occlusion by a physical structure in the environment). Individuals in groups with high tortuosity, and thus low net movement, persist for longer in such semi-occluded states and were particularly at risk. In direct contrast, prey in polarized groups were attacked less often, probably because their more directed movement gave less time for targeting to occur when they were within the high-risk area of the boundary. Further analysis of the pattern of risk seen in Fig. 2A demonstrates that although individuals in swarms are vulnerable at the boundaries, further from the boundary this effect weakens (fig. S6).
To ensure that our results were not sensitive to the distribution of prey types employed, and to demonstrate the selection of virtual prey strategies by real predators, we used the risk landscape in Fig. 2A to "evolve" our prey (23). We then presented either this evolved population or the original population to the fish in a second experiment (23). Although selection changed the frequencies of different prey types, and hence the group sizes and tortuosities in the projected simulation (table S2 and fig. S7), there was no evidence that the pattern observed in Fig. 2A changed between the pre- and postselection populations (population $\times$ attraction $\times$ orientation GLM: $\mathrm{LRT}_{1,18}=0.09, P=0.76$ ). Selection for orientation and attraction thus generalizes beyond an even distribution of behavioral parameters and appears relatively robust to frequency-dependent effects. Neither was there any evidence that selection had a detrimental effect on the fish's predatory behavior (table $\mathrm{S} 3$ ).

Our results show that predation risk is reduced among prey that exhibit both attraction and orientation under the conditions of our experiment, through an interaction between the confusion effect and the ability of prey to form coherent mobile groups. This is dependent on habitat properties that the predators exploit to facilitate targeting opportunities, without the necessity for prey individuals to react dynamically to the predator's presence, position, and/or attack $(6,18)$. The degree of control afforded by virtual prey populations, as developed here, could allow a closed feedback loop between predator attack and prey response to explore such properties. This may reveal further dependencies and synergies between anti-predatory adaptations, predator hunting strategies, and habitat variables $(2,19,27)$.
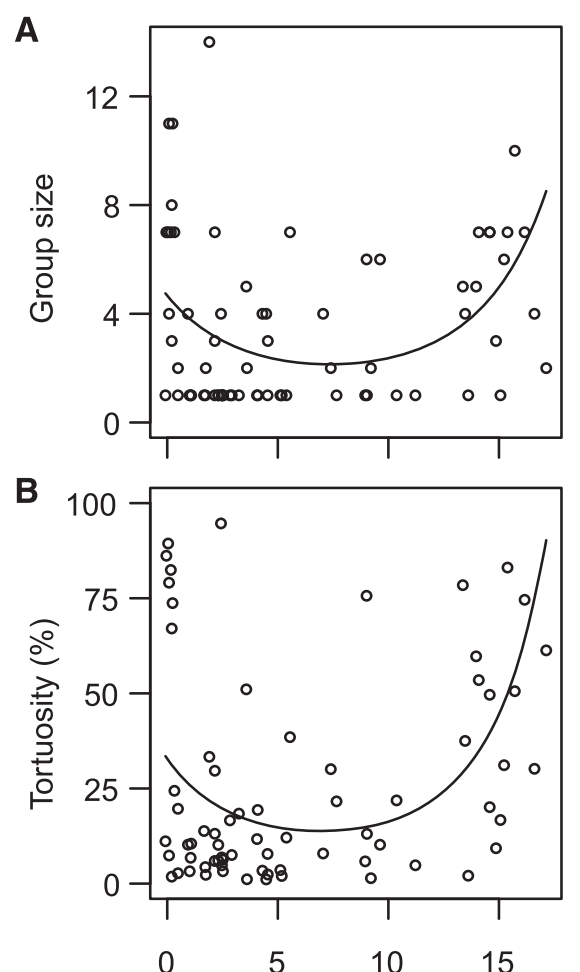

Height in vertical axis $(\mathrm{cm})$

Fig. 3. Preferential targeting of group size (A) and tortuosity (B) as a function of height in the vertical axis of the projection. The curves show fitted values from models with the (polynomial) vertical axis effect only, because the horizontal axis had no significant effect. Tortuosity is expressed as a percentage of the maximum possible value. The individual tortuosity of the target prey is shown here; a similar trend was found for the tortuosity of the target's group as a whole (GLM: LRT $2,65=14.89, P=$ 0.00059 ; group tortuosity $=1-$ group polarization). 


\section{References and Notes}

1. ]. Krause, G. D. Ruxton, Living in Groups (Oxford Univ. Press, Oxford, 2002).

2. W. Cresswell, J. L. Quinn, Oikos 104, 71 (2004).

3. W. L. Romey, A. R. Walston, P. J. Watt, Behav. Ecol. 19, 74 (2008).

4. W. D. Hamilton, J. Theor. Biol. 31, 295 (1971).

5. M. Ballerini et al., Proc. Natl. Acad. Sci. U.S.A. 105 1232 (2008).

6. N. O. Handegard et al., Curr. Biol. 22, 1213 (2012).

7. G. D. Ruxton, A. L. Jackson, C. R. Tosh, Behav. Ecol. 18 590 (2007).

8. K. A. Jones, A. L. Jackson, G. D. Ruxton, Behav. Ecol. 22, 831 (2011).

9. R. Lukeman, Y. X. Li, L. Edelstein-Keshet, Proc. Natl. Acad. Sci. U.S.A. 107, 12576 (2010)

10. Y. Katz, K. Tunstrøm, C. C. Ioannou, C. Huepe, I. D. Couzin, Proc. Natl. Acad. Sci. U.S.A. 108, 18720 (2011).

11. J. Buhl et al., Science 312, 1402 (2006).

12. I. D. Couzin, J. Krause, R. James, G. D. Ruxton N. R. Franks, J. Theor. Biol. 218, 1 (2002).

13. J. K. Parrish, L. Edelstein-Keshet, Science 284, 99 (1999)

14. S. Bazazi et al., Curr. Biol. 18, 735 (2008).
15. V. Guttal, I. D. Couzin, Proc. Natl. Acad. Sci. U.S.A. 107 16172 (2010).

16. D. Grünbaum, Evol. Ecol. 12, 503 (1998).

17. R. Vabø, L. Nøttestad, Fish. Oceanogr. 6, 155 (1997).

18. A. J. Wood, G. J. Ackland, Proc. Biol. Sci. 274, 1637 (2007).

19. T. J. Ehlinger, D. S. Wilson, Proc. Natl. Acad. Sci. U.S.A. 85, 1878 (1988).

20. H. Sayama, S. Dionne, C. Laramee, D. S. Wilson, in IEEE Symposium on Artificial Life (IEEE Conference Proceedings, Nashville, TN, 2009), pp. 85-91.

21. ]. E. Treherne, W. A. Foster, Anim. Behav, 30, 536 (1982).

22. W. Cresswell, Ibis 138, 684 (1996)

23. Materials and methods are available as supplementary materials on Science Online.

24. I. D. Couzin, ]. Krause, N. R. Franks, S. A. Levin, Nature 433, 513 (2005)

25. I. D. Couzin, ]. Krause, Adv. Stud. Behav. 32 1 (2003).

26. C. C. Ioannou, C. R. Tosh, L. Neville, ]. Krause, Behav. Ecol 19, 126 (2008)

27. C. R. Tosh, J. Theor. Biol. 281, 24 (2011).

Acknowledgments: We thank A. Hundal, M. Jiang, and

M. Singh for assistance and A. S. I. Wade, the Couzin lab, and three anonymous reviewers for comments on the manuscript. Funded by Office of Naval Research award N00014-09-1-1074, NSF award PHY-0848755, Searle Scholar award 08-SPP-201, and Army Research Office grant W911NG-11-1-0385 (I.D.C.); Defense Advanced Research Projects Agency grant HR0011-05-1-0057

(Princeton University); Leverhulme Trust Early Career Fellowship (C.C.I.); and a Ramalingaswami Fellowship from the Department of Biotechnology, Government of India (V.G.). All experiments were conducted in accordance with federal and state regulations and were approved by the Princeton University Institutional Animal Care and Use Committee. Data and code are freely available at http://icouzin. princeton.edu. 